\title{
Joint source and relay optimization for parallel MIMO relay networks
}

\author{
Apriana Toding, Muhammad RA Khandaker and Yue Rong*
}

\begin{abstract}
In this article, we study the optimal structure of the source precoding matrix and the relay amplifying matrices for multiple-input multiple-output (MIMO) relay communication systems with parallel relay nodes. Two types of receivers are considered at the destination node: (1) The linear minimal mean-squared error (MMSE) receiver; (2) The nonlinear decision feedback equalizer based on the minimal MSE criterion. We show that for both receiver schemes, the optimal source precoding matrix and the optimal relay amplifying matrices have a beamforming structure. Using such optimal structure, joint source and relay power loading algorithms are developed to minimize the MSE of the signal waveform estimation at the destination. Compared with existing algorithms for parallel MIMO relay networks, the proposed joint source and relay beamforming algorithms have significant improvement in the system bit-error-rate performance.
\end{abstract}

Keywords: MIMO relay, Parallel relay network, Beamforming, DFE, Non-regenerative relay

\section{Introduction}

Recently, multiple-input multiple-output (MIMO) relay communication systems have attracted much research interest [1-10]. Many studies have studied the optimal relay amplifying matrix for the source-relay-destination channel. In $[2,3]$, the optimal relay amplifying matrix maximizing the mutual information (MI) between the source and destination was derived assuming that the source covariance matrix is an identity matrix. In [4-6], the relay amplifying matrix was designed to minimize the meansquared error (MSE) of the signal waveform estimation at the destination.

A few research has studied the jointly optimal structure of the source precoding matrix and the relay amplifying matrix. In [7], both the source and relay matrices were jointly designed to maximize the source-destination MI. A unified framework was developed in $[8,9]$ to jointly optimize the source and relay matrices for a broad class of objective functions. All the works in [2-9] considered a single relay node at each hop. The authors of [10] investigated the optimal relay amplifying matrices for two-hop MIMO relay networks with multiple parallel relay nodes. However, the source precoding matrix was not optimized

*Correspondence:y.rong@curtin.edu.au

Department of Electrical and Computer Engineering, Curtin University, Bentley, WA 6102, Australia in [10]. In [11,12], parallel MIMO relay systems have been investigated with power constraint at the output of the second-hop channel considering a linear and a nonlinear receiver, respectively.

In this article, we jointly optimize the source precoding matrix and relay amplifying matrices for a two-hop MIMO relay network with multiple parallel relay nodes and transmission power constrain at each relay node. Two types of receivers are considered at the destination node: (1) The linear minimal MSE (MMSE) receiver; (2) The nonlinear decision feedback equalizer (DFE) based on the MMSE criterion. We show that for both receiver schemes, the optimal source precoding matrix and the optimal relay amplifying matrices have a beamforming structure. This result generalizes the optimal source and relay matrices design from a single relay node per hop case $[8,13]$ to multiple parallel relay nodes scenario. Simulation results demonstrate that with a linear MMSE receiver at the destination, the system with the jointly optimal source and relay matrices has a better bit-error-rate (BER) performance compared with that of the relay system with only optimal relay matrices developed in [10]. Moreover, a nonlinear DFE receiver recovers the source signals successively by exploiting the finite alphabet property of the source signals. Using a DFE receiver we can remove the effect of interferences of the data streams we have

\section{是 Springer}

(c) 2012 Toding et al. licensee Springer. This is an Open Access article distributed under the terms of the Creative Commons Attribution License (http://creativecommons.org/licenses/by/2.0), which permits unrestricted use, distribution, and reproduction in any medium, provided the original work is properly cited. 
already recovered from the subsequent streams. Therefore, introducing a nonlinear MMSE-DFE receiver at the destination yields further improvement in the system BER performance compared with the MIMO parallel relay system using a linear MMSE receiver. Our simulation results also demonstrate a better performance of the nonlinear receiver algorithm.

The rest of this article is organized as follows. In the following section, we introduce the model of parallel MIMO relay systems with a linear MMSE receiver and a nonlinear MMSE-DFE receiver at the destination. In Section "MMSE relay design" we study the optimal structure of the source and relay matrices using both receiver schemes, after that simulation results are given in Section "Simulations". Finally, conclusions are drawn in the last section.

\section{System model}

Figure 1 illustrates a two-hop MIMO relay communication system consisting of one source node, $K$ parallel relay nodes, and one destination node. We assume that the source and the destination nodes have $N_{s}$ and $N_{d}$ antennas, respectively, and each relay node has $N_{r}$ antennas. The generalization to the system with different number of antennas at each relay node is straightforward. Due to its merit of simplicity, we consider the amplify-and-forward relaying scheme at each relay. The communication process between the source and destination nodes is completed in two time slots. In the first time slot, the $N_{b} \times 1$ modulated source symbol vector $\mathbf{s}$ is linearly precoded as

$$
\mathbf{x}=\mathbf{B} \mathbf{s}
$$

where $\mathrm{B}$ is an $N_{s} \times N_{b}$ source precoding matrix. We assume that the source signal vector satisfies $\mathrm{E}\left[\mathbf{s s}^{H}\right]=$ $\mathbf{I}_{N_{b}}$, where $\mathbf{I}_{n}$ stands for an $n \times n$ identity matrix, $(\cdot)^{H}$ is the matrix (vector) Hermitian transpose, and E[ . ] denotes statistical expectation. The precoded vector $\mathbf{x}$ is transmitted to $K$ parallel relay nodes. The $N_{r} \times 1$ received signal vector at the $i$ th relay node can be written as

$$
\mathbf{y}_{r, i}=\mathbf{H}_{s r, i} \mathbf{x}+\mathbf{v}_{r, i}, \quad i=1, \ldots, K
$$

where $\mathbf{H}_{s r, i}$ is the $N_{r} \times N_{s}$ MIMO channel matrix between the source and the $i$ th relay nodes and $\mathbf{v}_{r, i}$ is the additive Gaussian noise vector at the $i$ th relay node.

In the second time slot, the source node is silent, while each relay node transmits the linearly amplified signal vector to the destination node as

$$
\mathbf{x}_{r, i}=\mathbf{F}_{i} \mathbf{y}_{r, i}, \quad i=1, \ldots, K
$$

where $\mathbf{F}_{i}$ is the $N_{r} \times N_{r}$ amplifying matrix at the $i$ th relay node. The received signal vector at the destination node can be written as

$$
\mathbf{y}_{d}=\sum_{i=1}^{K} \mathbf{H}_{r d, i} \mathbf{x}_{r, i}+\mathbf{v}_{d}
$$

where $\mathbf{H}_{r d, i}$ is the $N_{d} \times N_{r}$ MIMO channel matrix between the $i$ th relay and the destination nodes, $\mathbf{v}_{d}$ is the additive Gaussian noise vector at the destination node.

Substituting (1)-(3) into (4), we have

$$
\begin{aligned}
\mathbf{y}_{d} & =\sum_{i=1}^{K}\left(\mathbf{H}_{r d, i} \mathbf{F}_{i} \mathbf{H}_{s r, i} \mathbf{B} \mathbf{s}+\mathbf{H}_{r d, i} \mathbf{F}_{i} \mathbf{v}_{r, i}\right)+\mathbf{v}_{d} \\
& =\mathbf{H}_{r d} \mathbf{F} \mathbf{H}_{s r} \mathbf{B} \mathbf{s}+\mathbf{H}_{r d} \mathbf{F}_{r}+\mathbf{v}_{d}
\end{aligned}
$$

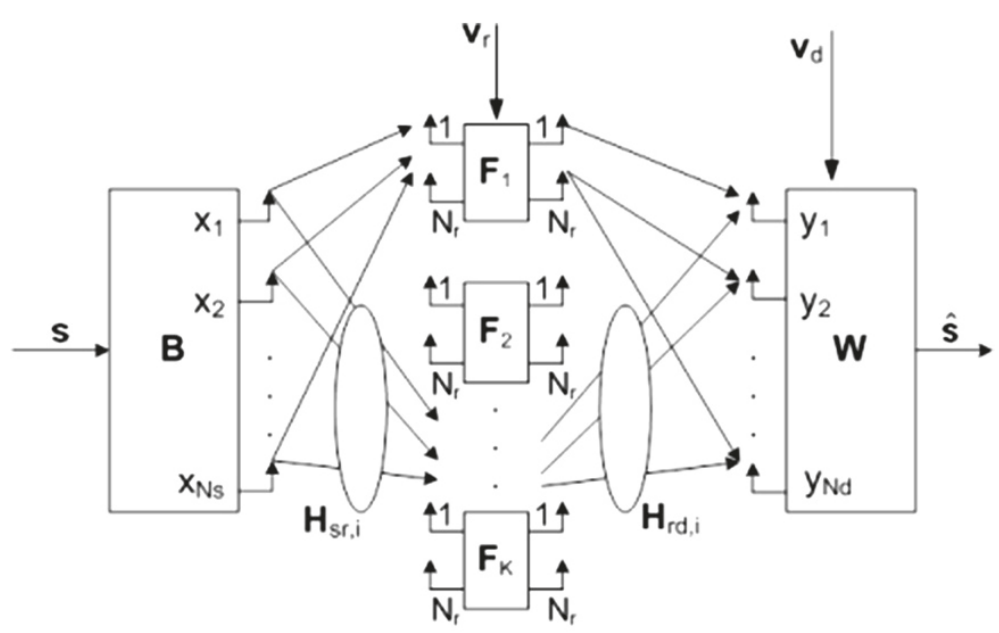

Source

Relay

Destination

Figure 1 Block diagram of a parallel MIMO relay communication system. 
where we define

$$
\begin{aligned}
\mathbf{H}_{s r} & \triangleq\left[\mathbf{H}_{s r, 1}^{T}, \mathbf{H}_{s r, 2}^{T}, \ldots, \mathbf{H}_{s r, K}^{T}\right]^{T} \\
\mathbf{H}_{r d} & \triangleq\left[\mathbf{H}_{r d, 1}, \mathbf{H}_{r d, 2}, \ldots, \mathbf{H}_{r d, K}\right] \\
\mathbf{F} & \triangleq \mathrm{bd}\left[\mathbf{F}_{1}, \mathbf{F}_{2}, \ldots, \mathbf{F}_{K}\right] \\
\mathbf{v}_{r} & \triangleq\left[\mathbf{v}_{r, 1}^{T}, \mathbf{v}_{r, 2}^{T}, \ldots, \mathbf{v}_{r, K}^{T}\right]^{T} .
\end{aligned}
$$

Here $(\cdot)^{T}$ denotes the matrix (vector) transpose, bd $[\cdot]$ stands for a block-diagonal matrix, $\mathbf{H}_{s r}$ is a $K N_{r} \times N_{s}$ channel matrix between the source node and all $K$ relay nodes, $\mathbf{H}_{r d}$ is an $N_{d} \times K N_{r}$ channel matrix between all relay nodes and the destination node, $\mathbf{v}_{r}$ is obtained by stacking the noise vectors at all relays and $\mathbf{F}$ is the $K N_{r} \times K N_{r}$ equivalent block diagonal relay amplifying matrix. The diagram of the equivalent MIMO relay system described by (5) is shown in Figure 2 (without the receiving filters). We assume that all noises are independent and identically distributed (i.i.d.) Gaussian noise with zero mean and unit variance.

By introducing

$$
\overline{\mathbf{F}} \triangleq \mathbf{H}_{r d} \mathbf{F}
$$

the received signal vector at the destination can equivalently be written as

$$
\mathbf{y}_{d}=\overline{\mathbf{F}} \mathbf{H}_{s r} \mathbf{B s}+\overline{\mathbf{F}} \mathbf{v}_{r}+\mathbf{v}_{d}=\overline{\mathbf{H}} \mathbf{s}+\overline{\mathbf{v}}
$$

where we define $\overline{\mathbf{H}} \triangleq \overline{\mathbf{F}} \mathbf{H}_{s r} \mathbf{B}$ as the effective MIMO channel matrix of the source-relay-destination link, and $\overline{\mathbf{v}} \triangleq$ $\overline{\mathbf{F}} \mathbf{v}_{r}+\mathbf{v}_{d}$ as the equivalent noise vector. The transmission power consumed by each relay node can be expressed as

$$
\begin{aligned}
\mathrm{E}\left[\operatorname{tr}\left(\mathbf{x}_{r, i} \mathbf{x}_{r, i}^{H}\right)\right] & =\operatorname{tr}\left(\mathbf{F}_{i}\left[\mathbf{H}_{s r, i} \mathbf{B} \mathbf{B}^{H^{H}} \mathbf{H}_{s r, i}^{H}+\mathbf{I}_{N_{r}}\right] \mathbf{F}_{i}^{H}\right), \\
i & =1, \ldots, K
\end{aligned}
$$

where $\operatorname{tr}(\cdot)$ stands for the matrix trace. In the following, we introduce the linear MMSE receiver and the nonlinear MMSE-DFE receiver for MIMO relay systems.

\section{Linear MMSE receiver}

Using a linear receiver, the estimated signal waveform vector at the destination node in Figure 2 (without the feedback operation) is given by $\hat{\mathbf{s}}=\mathbf{W}^{H} \mathbf{y}_{d}$, where $\mathbf{W}$ is an $N_{d} \times N_{b}$ weight matrix. The MSE of the signal waveform estimation is given by

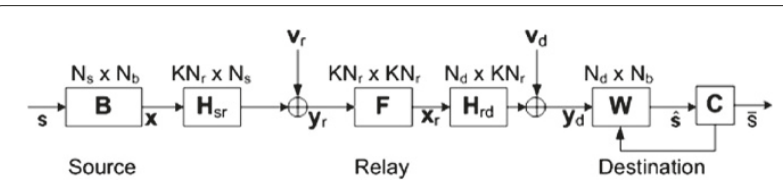

Figure 2 Block diagram of the equivalent MIMO relay system.

$$
\begin{aligned}
\mathrm{MSE} & =\operatorname{tr}\left(\mathrm{E}\left[(\hat{\mathbf{s}}-\mathbf{s})(\hat{\mathbf{s}}-\mathbf{s})^{H}\right]\right) \\
& =\operatorname{tr}\left(\left(\mathbf{W}^{H} \overline{\mathbf{H}}-\mathbf{I}_{N_{b}}\right)\left(\mathbf{W}^{H} \overline{\mathbf{H}}-\mathbf{I}_{N_{b}}\right)^{H}+\mathbf{W}^{H} \mathbf{C}_{\overline{\mathbf{v}}} \mathbf{W}\right)
\end{aligned}
$$

where $\mathbf{C}_{\bar{v}}$ is the equivalent noise covariance matrix given by $\mathbf{C}_{\overline{\boldsymbol{v}}}=\mathrm{E}\left[\overline{\mathbf{v}}^{H}\right]=\overline{\mathbf{F}} \overline{\mathbf{F}}^{H}+\mathbf{I}_{N_{d}}$. The weight matrix $\mathbf{W}$ which minimizes (8) is the Wiener filter and can be written as

$$
\mathbf{W}=\left(\overline{\mathbf{H}} \overline{\mathbf{H}}^{H}+\mathbf{C}_{\bar{v}}\right)^{-1} \overline{\mathbf{H}}
$$

where $(\cdot)^{-1}$ denotes the matrix inversion. Substituting (9) back into (8), it can be seen that the MSE is a function of $\overline{\mathbf{F}}$ and $\mathbf{B}$ and can be written as

$$
\mathrm{MSE}=\operatorname{tr}\left(\left[\mathbf{I}_{N_{b}}+\overline{\mathbf{H}}^{H} \mathbf{C}_{\bar{v}}^{-1} \overline{\mathbf{H}}\right]^{-1}\right) .
$$

\section{Nonlinear MMSE-DFE receiver}

With a nonlinear DFE receiver employed at the destination node, the source symbols are detected successively with the $N_{b}$ th symbol detected first and the first symbol detected last. The equivalent MIMO relay system model is shown in Figure 2. Assuming that there is no error propagation in the DFE receiver, the estimated source symbol vector is

$$
\overline{\mathbf{s}}=\overline{\mathbf{W}}^{H} \mathbf{y}_{d}-\mathbf{C s}=\left(\overline{\mathbf{W}}^{H} \overline{\mathbf{H}}-\mathbf{C}\right) \mathbf{s}+\overline{\mathbf{W}}^{H} \overline{\mathbf{v}}
$$

where $\overline{\mathbf{W}}$ is the $N_{d} \times N_{b}$ feed-forward weight matrix, $\mathbf{C}$ is the $N_{b} \times N_{b}$ strictly upper-triangle feedback matrix of the DFE receiver. To minimize the error of the signal estimation in (11), we have $\mathbf{C}=\mathcal{U}\left[\overline{\mathbf{W}}^{H} \overline{\mathbf{H}}\right]$, where $\mathcal{U}\left[\overline{\mathbf{W}}^{H} \overline{\mathbf{H}}\right]$ denotes the strictly upper-triangular part of $\overline{\mathbf{W}}^{H} \overline{\mathbf{H}}$.

When the MMSE criterion is used to estimate each symbol, the feed-forward matrix $\overline{\mathbf{W}}$ is given as

$$
[\overline{\mathbf{W}}]_{k}=\left([\overline{\mathbf{H}}]_{1: k}[\overline{\mathbf{H}}]_{1: k}^{H}+\mathbf{C}_{\bar{v}}\right)^{-1}[\overline{\mathbf{H}}]_{k}, \quad k=1, \ldots, N_{b}
$$

where $[\mathbf{A}]_{1: k}$ stands for a matrix containing the first $k$ columns of $\mathbf{A}$, and $[\mathbf{A}]_{k}$ is the $k$ th column of $\mathbf{A}$. Let us now introduce the following $\mathrm{QR}$ decomposition

$$
\mathbf{G} \triangleq\left[\begin{array}{l}
\mathbf{C}_{\bar{v}}^{-\frac{1}{2}} \overline{\mathbf{H}} \\
\mathbf{I}_{N_{b}}
\end{array}\right]=\mathbf{Q R}=\left[\begin{array}{l}
\overline{\mathbf{Q}} \\
\mathbf{Q}
\end{array}\right] \mathbf{R}
$$

where $\mathbf{R}$ is an $N_{b} \times N_{b}$ upper-triangular matrix with all positive diagonal elements, $\mathbf{Q}$ is an $\left(N_{d}+N_{b}\right) \times N_{b}$ semi-unitary matrix with $\mathbf{Q}^{H} \mathbf{Q}=\mathbf{I}_{N_{b}}, \overline{\mathbf{Q}}$ is a matrix containing the first $N_{d}$ rows of $\mathbf{Q}$, and $\mathbf{Q}$ contains the last $N_{b}$ rows of $\mathbf{Q}$.

Using the QR decomposition (12), it has been shown in [13] that the feed-forward weight matrix $\overline{\mathbf{W}}$, the feedback matrix $\mathbf{C}$, and the MSE matrix $\mathbf{E}=\mathrm{E}\left[(\overline{\mathbf{s}}-\mathbf{s})(\overline{\mathbf{s}}-\mathbf{s})^{H}\right]$ can be represented as

$$
\overline{\mathbf{W}}=\mathbf{C}_{\bar{v}}^{-\frac{1}{2}} \overline{\mathbf{Q}} \mathbf{D}_{R}^{-1}, \quad \mathbf{C}=\mathbf{D}_{R}^{-1} \mathbf{R}-\mathbf{I}_{N_{b}}, \quad \mathbf{E}=\mathbf{D}_{R}^{-2}
$$


where $\mathbf{D}_{R}$ is a matrix taking the diagonal elements of $\mathbf{R}$ as the main diagonal and zero elsewhere.

\section{Minimal MMSE relay design}

In this section, we address the joint source and relay optimization problem for systems with a linear MMSE receiver and a nonlinear MMSE-DFE receiver at the destination node, respectively. In particular, we show that for both receiver schemes, the optimal source and relay matrices have a general beamforming structure.

\section{Optimal design with linear MMSE receiver}

Based on (7) and (10), the joint source and relay optimization problem with a linear MMSE receiver used at the destination node can be formulated as

$$
\begin{aligned}
\min _{\left\{\mathbf{F}_{i}\right\}, \mathbf{B}} & \operatorname{tr}\left(\left[\mathbf{I}_{N_{b}}+\overline{\mathbf{H}}^{H} \mathbf{C}_{\bar{v}}^{-1} \overline{\mathbf{H}}\right]^{-1}\right) \\
\text { s.t. } & \operatorname{tr}\left(\mathbf{B B}^{H}\right) \leq P_{s} \\
& \operatorname{tr}\left(\mathbf{F}_{i}\left[\mathbf{H}_{s r, i} \mathbf{B} \mathbf{B}^{H} \mathbf{H}_{s r, i}^{H}+\mathbf{I}_{N_{r}}\right] \mathbf{F}_{i}^{H}\right) \leq P_{x, i}, \quad i=1, \ldots, K
\end{aligned}
$$

where (15) is the transmit power constraint at the source node, while (16) is the power constraint at each relay node. Here $P_{s}>0$ and $P_{x, i}>0, i=1, \ldots, K$, are the corresponding power budget. Obviously, to avoid any loss of transmission power in the relay system when a linear receiver is used, there should be $N_{b} \leq \min \left(N_{s}, K N_{r}, N_{d}\right)$.

Due to the power constraint at each relay node (16), the source and relay matrices optimization problem (14)-(16) is much more challenging to solve when $K \geq 2$ compared with the case of $K=1$. To overcome this difficulty, we relax the power constraints in (16) by considering the power of the signal at the output of $\mathbf{H}_{r d}$, which can be expressed as [10]

$$
\begin{aligned}
\mathrm{E}\left[\operatorname{tr}\left(\left(\mathbf{H}_{r d} \mathbf{x}_{r}\right)\left(\mathbf{H}_{r d} \mathbf{x}_{r}\right)^{H}\right)\right] & =\operatorname{tr}\left(\overline{\mathbf{F}}\left[\mathbf{H}_{s r} \mathbf{B} \mathbf{B}^{H} \mathbf{H}_{s r}^{H}+\mathbf{I}_{K N_{r}}\right] \overline{\mathbf{F}}^{H}\right) \\
& \leq P_{x} \operatorname{tr}\left(\mathbf{H}_{r d} \mathbf{H}_{r d}^{H}\right) .
\end{aligned}
$$

Here, $P_{x} \triangleq \sum_{i=1}^{K} P_{x, i}$ is the total transmission power budget available to all $K$ relay nodes. Using (17), the relaxed joint source and relay optimization problem can be written as

$$
\begin{array}{ll}
\min _{\overline{\mathbf{F}}, \mathbf{B}} & \operatorname{tr}\left(\left[\mathbf{I}_{N_{b}}+\overline{\mathbf{H}}^{H} \mathbf{C}_{\bar{v}}^{-1} \overline{\mathbf{H}}\right]^{-1}\right) \\
\text { s.t. } & \operatorname{tr}\left(\mathbf{B} \mathbf{B}^{H}\right) \leq P_{s} \\
& \operatorname{tr}\left(\overline{\mathbf{F}}\left[\mathbf{H}_{s r} \mathbf{B} \mathbf{B}^{H} \mathbf{H}_{s r}^{H}+\mathbf{I}_{K N_{r}}\right] \overline{\mathbf{F}}^{H}\right) \leq P_{r}
\end{array}
$$

where $P_{r} \triangleq P_{x} \operatorname{tr}\left(\mathbf{H}_{r d} \mathbf{H}_{r d}^{H}\right)$.

Let $\mathbf{H}_{s r}=\mathbf{U}_{s} \boldsymbol{\Lambda}_{s} \mathbf{V}_{s}^{H}$ denote the singular value decomposition (SVD) of $\mathbf{H}_{s r}$, where the dimensions of $\mathbf{U}_{s}, \boldsymbol{\Lambda}_{s}, \mathbf{V}_{s}$ are
$K N_{r} \times K N_{r}, K N_{r} \times N_{s}, N_{s} \times N_{s}$, respectively. We assume that the main diagonal elements of $\boldsymbol{\Lambda}_{s}$ are arranged in a decreasing order. The optimal structure of $\overline{\mathbf{F}}$ and $\mathbf{B}$ as the solution to the problem (18)-(20) is given by

$$
\overline{\mathbf{F}}=\mathbf{V} \boldsymbol{\Lambda}_{f} \mathbf{U}_{s, 1}^{H}, \quad \mathbf{B}=\mathbf{V}_{s, 1} \boldsymbol{\Lambda}_{b}
$$

where $\mathbf{V}$ is any $N_{d} \times N_{b}$ semi-unitary matrix with $\mathbf{V}^{H} \mathbf{V}=$ $\mathbf{I}_{N_{b}}, \mathbf{U}_{s, 1}$ and $\mathbf{V}_{s, 1}$ contain the leftmost $N_{b}$ columns of $\mathbf{U}_{s}$ and $\mathbf{V}_{s}$, respectively, $\boldsymbol{\Lambda}_{f}$ and $\boldsymbol{\Lambda}_{b}$ are $N_{b} \times N_{b}$ diagonal matrices. The proof of (21) is similar to the proof of Theorem 1 in [8]. From (21), we see that the optimal $\overline{\mathbf{F}}$ and $\mathbf{B}$ have a beamforming structure. In fact, they jointly diagonalize the source-relay-destination channel $\overline{\mathbf{H}}$ up to a rotation matrix $\mathbf{V}$. Using (21), the joint source-relay optimization problem (18)-(20) becomes

$$
\begin{aligned}
\min _{\boldsymbol{\Lambda}_{f}, \boldsymbol{\Lambda}_{b}} & \operatorname{tr}\left(\left[\mathbf{I}_{N_{b}}+\left(\boldsymbol{\Lambda}_{f} \boldsymbol{\Lambda}_{s} \boldsymbol{\Lambda}_{b}\right)^{2}\left(\boldsymbol{\Lambda}_{f}^{2}+\mathbf{I}_{N_{b}}\right)^{-1}\right]^{-1}\right) \\
\text { s.t. } & \operatorname{tr}\left(\boldsymbol{\Lambda}_{b}^{2}\right) \leq P_{s} \\
& \operatorname{tr}\left(\boldsymbol{\Lambda}_{f}^{2}\left[\left(\boldsymbol{\Lambda}_{s} \boldsymbol{\Lambda}_{b}\right)^{2}+\mathbf{I}_{N_{b}}\right]\right) \leq P_{r} .
\end{aligned}
$$

Let us denote $\lambda_{f, i}, \lambda_{s, i}, \lambda_{b, i}, i=1, \ldots, N_{b}$, as the main diagonal elements of $\boldsymbol{\Lambda}_{f}, \boldsymbol{\Lambda}_{s}, \boldsymbol{\Lambda}_{b}$, respectively, and introduce

$$
\begin{aligned}
a_{i} & \triangleq \lambda_{s, i}^{2}, \quad x_{i} \triangleq \lambda_{b, i}^{2}, \quad y_{i} \triangleq \lambda_{f, i}^{2}\left[\left(\lambda_{s, i} \lambda_{b, i}\right)^{2}+1\right], \\
i & =1, \ldots, N_{b}
\end{aligned}
$$

The optimization problem (22)-(24) can be equivalently rewritten as

$$
\begin{aligned}
\min _{\mathbf{x}, \mathbf{y}} & \sum_{i=1}^{N_{b}} \frac{a_{i} x_{i}+y_{i}+1}{a_{i} x_{i} y_{i}+a_{i} x_{i}+y_{i}+1} \\
\text { s.t. } & \sum_{i=1}^{N_{b}} x_{i} \leq P_{s}, \quad x_{i} \geq 0, \quad i=1, \ldots, N_{b} \\
& \sum_{i=1}^{N_{b}} y_{i} \leq P_{r}, \quad y_{i} \geq 0, \quad i=1, \ldots, N_{b}
\end{aligned}
$$

where $\mathbf{x} \triangleq\left[x_{1}, x_{2}, \ldots, x_{N_{b}}\right]^{T}$ and $\mathbf{y} \triangleq\left[y_{1}, y_{2}, \ldots, y_{N_{b}}\right]^{T}$. The problem (26)-(28) can be solved by an iterative method developed in [8], where in each iteration, $\mathbf{x}$ and $\mathbf{y}$ are updated alternatingly by fixing the other vector. After the optimal $\mathbf{x}$ and $\mathbf{y}$ are found, $\lambda_{f, i}$ and $\lambda_{b, i}$ can be obtained from (25) as

$$
\lambda_{f, i}=\sqrt{\frac{y_{i}}{\lambda_{s, i}^{2} x_{i}+1}}, \quad \lambda_{b, i}=\sqrt{x_{i}}, \quad i=1, \ldots, N_{b} .
$$

Using (6) and the optimal structure of $\overline{\mathbf{F}}$ and $\mathbf{B}$ in (21), we have $\mathbf{H}_{r d, i} \mathbf{F}_{i}=\mathbf{V} \boldsymbol{\Lambda}_{f} \boldsymbol{\Phi}_{i}$, where matrix $\boldsymbol{\Phi}_{i}$ contains the $(i-1) N_{r}+1$ to $i N_{r}$ columns of $\mathbf{U}_{s, 1}^{H}$. Then we obtain 


$$
\mathbf{F}_{i}=\mathbf{H}_{r d, i}^{\dagger} \mathbf{V} \boldsymbol{\Lambda}_{f} \boldsymbol{\Phi}_{i}, \quad i=1, \ldots, K
$$

where $(\cdot)^{\dagger}$ denotes matrix pseudo-inverse. Finally, we scale $\mathbf{F}_{i}$ in (30) to satisfy the power constraint (16) at each relay node as

$$
\tilde{\mathbf{F}}_{i}=\alpha_{i} \mathbf{F}_{i}, \quad i=1, \ldots, K
$$

where the scaling factor $\alpha_{i}$ is given by

$$
\alpha_{i}=\sqrt{\frac{P_{x, i}}{\operatorname{tr}\left(\mathbf{F}_{i}\left[\mathbf{H}_{s r, i} \mathbf{B} \mathbf{B}^{H} \mathbf{H}_{s r, i}^{H}+\mathbf{I}_{N_{r}}\right] \mathbf{F}_{i}^{H}\right)}}, \quad i=1, \ldots, K .
$$

\section{Optimal design with nonlinear MMSE-DFE receiver}

Using (12), (13), and the relaxed power constraint (20), the joint source and relay optimization problem which minimizes the MSE of the signal waveform estimation with a nonlinear MMSE-DFE receiver can be formulated as

$$
\begin{array}{cl}
\min _{\overline{\mathbf{F}}, \mathbf{B}} & \operatorname{tr}\left(\mathbf{D}_{R}^{-2}\right) \\
\text { s.t. } & \mathbf{G}=\mathbf{Q R} \\
& \operatorname{tr}\left(\mathbf{B B}^{H}\right) \leq P_{s} \\
& \operatorname{tr}\left(\overline{\mathbf{F}}\left[\mathbf{H}_{s r} \mathbf{B} \mathbf{B}^{H} \mathbf{H}_{s r}^{H}+\mathbf{I}_{K N_{r}}\right] \overline{\mathbf{F}}^{H}\right) \leq P_{r} .
\end{array}
$$

Let us introduce $M \triangleq \min \left(N_{b}, \operatorname{rank}\left(\mathbf{H}_{s r}\right)\right)$, where $\operatorname{rank}(\cdot)$ denotes the rank of a matrix. The optimal source precoding matrix and the optimal relay amplifying matrices as the solution to the problem (33)-(36) are given by

$$
\overline{\mathbf{F}}=\mathbf{U} \boldsymbol{\Delta}_{f} \mathbf{U}_{s, 1}^{H}, \quad \mathbf{B}=\mathbf{V}_{s, 1} \boldsymbol{\Delta}_{b} \mathbf{V}_{r}^{H}
$$

where $\boldsymbol{\Delta}_{f}$ and $\boldsymbol{\Delta}_{b}$ are $M \times M$ diagonal matrices, $\mathbf{U}$ is any $N_{d} \times M$ semi-unitary matrix with $\mathbf{U}^{H} \mathbf{U}=\mathbf{I}_{M}, \mathbf{U}_{s, 1}$ and $\mathbf{V}_{s, 1}$ contain the leftmost $M$ vectors of $\mathbf{U}_{s}$ and $\mathbf{V}_{s}$, respectively, and $\mathbf{V}_{r}$ is an $N_{b} \times M$ semi-unitary matrix $\left(\mathbf{V}_{r}^{H} \mathbf{V}_{r}=\mathbf{I}_{M}\right)$ such that the QR decomposition in (34) holds. The proof of (37) is similar to the proof of Theorem 2 in [13].

From (37), we find that both $\overline{\mathbf{F}}$ and $\mathbf{B}$ have a beamforming structure. In particular, they jointly diagonalize the source-relay-destination channel matrix $\overline{\mathbf{H}}$ up to rotation matrices $\mathbf{U}$ and $\mathbf{V}_{r}$. It can be shown similar to $[13,14]$ that the constraint (34) is equivalent to

$$
\mathbf{d}\left[\mathbf{D}_{R}\right] \prec \sigma_{G}
$$

where $\prec$ stands for multiplicative majorization [15], $\sigma_{G}$ is a column vector containing all singular values of $\mathbf{G}$, and $\mathbf{d}\left[\mathbf{D}_{R}\right]$ is a column vector containing all diagonal elements of $\mathbf{D}_{R}$. Using (37) and (38), the optimization problem (33)(36) can equivalently be rewritten as

$$
\begin{array}{ll}
\min _{\delta_{f}, \delta_{b}} & \operatorname{tr}\left(\mathbf{D}_{R}^{-2}\right) \\
\text { s.t. } & \mathbf{d}\left[\mathbf{D}_{R}^{2}\right] \prec_{\mathrm{w}}\left[\left\{1+\frac{\left(\delta_{f, i} \lambda_{s, i} \delta_{b, i}\right)^{2}}{\delta_{f, i}^{2}+1}\right\}^{T}, \mathbf{1}_{N_{b}-M}\right]^{T} \\
& \sum_{i=1}^{M} \delta_{b, i}^{2} \leq P_{s} \\
& \sum_{i=1}^{M} \delta_{f, i}^{2}\left[\left(\lambda_{s, i} \delta_{b, i}\right)^{2}+1\right] \leq P_{r} \\
& \delta_{b, i} \geq 0, \quad \delta_{f, i} \geq 0, \quad i=1, \ldots, M
\end{array}
$$

where $\prec_{\mathrm{w}}$ stands for weakly multiplicative submajorization [15], $\mathbf{1}_{N_{b}-M}$ denotes a $1 \times\left(N_{b}-M\right)$ vector with all 1 elements, $\boldsymbol{\delta}_{f} \triangleq\left[\delta_{f, 1}, \delta_{f, 2}, \ldots, \delta_{f, M}\right]$, and $\boldsymbol{\delta}_{b} \triangleq$ $\left[\delta_{b, 1}, \delta_{b, 2}, \ldots, \delta_{b, M}\right]$.

Using the definition of the operator $\prec_{\mathrm{w}}$ in [15] and the notations of

$$
\begin{aligned}
& a_{i} \triangleq \lambda_{s, i}^{2}, \quad \tilde{x}_{i} \triangleq \delta_{b, i}^{2}, \quad \tilde{y}_{i} \triangleq \delta_{f, i}^{2}\left[\left(\lambda_{s, i} \delta_{b, i}\right)^{2}+1\right], \\
& i=1, \ldots, M
\end{aligned}
$$

the optimization problem (39)-(43) can equivalently be converted to the following problem

$$
\begin{aligned}
\min _{\tilde{\mathbf{x}}, \tilde{\mathbf{y}}} & \sum_{i=1}^{M} \log \frac{a_{i} \tilde{x}_{i}+\tilde{y}_{i}+1}{a_{i} \tilde{x}_{i} \tilde{y}_{i}+a_{i} \tilde{x}_{i}+\tilde{y}_{i}+1} \\
\text { s.t. } & \sum_{i=1}^{M} \tilde{x}_{i} \leq P_{s}, \quad \tilde{x}_{i} \geq 0, \quad i=1, \ldots, M \\
& \sum_{i=1}^{M} \tilde{y}_{i} \leq P_{r}, \quad \tilde{y}_{i} \geq 0, \quad i=1, \ldots, M .
\end{aligned}
$$

Similar to the problem (26)-(28), the problem (45)-(47) can be solved by an iterative method developed in [8]. Then $\mathbf{F}_{i}, i=1, \ldots, K$, are obtained similar to (29) and (30). Finally, the relay matrices satisfying the constraints (16) are obtained as (31) and (32).

The major computation task of the proposed algorithms lies in performing the SVD of channel matrices and calculating the power loading parameters. Since both algorithms require the same amount of channel information at each node and use iterative approach to obtain the optimal power allocation vectors, they have the same computational complexity order. It can easily be seen from (26)-(28) that the computational complexity of the proposed algorithms is the same as an iterative water-filling algorithm [8] with two variables of dimension $N_{b} \times 1$. 


\section{Simulations}

In this section, we study the performance of the proposed jointly optimal source and relay beamforming algorithms for parallel MIMO relay systems with linear MMSE and nonlinear MMSE-DFE receivers, respectively. All simulations are conducted in a flat Rayleigh fading environment where the channel matrices have zero-mean entries with variances $\sigma_{s}^{2} / N_{s}$ and $\sigma_{r}^{2} /\left(K N_{r}\right)$ for $\mathbf{H}_{s r}$ and $\mathbf{H}_{r d}$, respectively. The BPSK constellations are used to modulate the source symbols, and all noises are i.i.d. Gaussian with zero mean and unit variance. We define $\mathrm{SNR}_{\mathrm{s}}=\sigma_{s}^{2} P_{s} K N_{r} / N_{s}$ and $\mathrm{SNR}_{\mathrm{r}}=\sigma_{r}^{2} P_{r} N_{d} /\left(K N_{r}\right)$ as the signal-to-noise ratio (SNR) for the source-relay link and the relay-destination link, respectively. In all simulations, we set $N_{b}=N_{s}=$ $N_{r}=N_{d}=3$ and $\mathrm{SNR}_{\mathrm{r}}=20 \mathrm{~dB}$. We transmit $1000 N_{s}$ randomly generated bits in each channel realization, and all simulation results are averaged over 200 channel realizations.

In the first example, a parallel MIMO relay system with $K=3$ relay nodes is simulated. We compare the BER performance of the following algorithms: (i) two proposed joint source and relay schemes considering individual power constraints (IPC) at each relay node; (ii) The source and relay matrices design in $[11,12]$ with power constraint at the output of $\mathbf{H}_{r d}$; (iii) the naive amplify-and-forward (NAF) algorithm where both the source and relay matrices are scaled identity matrices satisfying power constraints (19) and (20); (iv) the optimal relay only (ORO) algorithm developed in [10] where the relay matrices are optimized based on the MMSE criterion, while the source precoding matrix is a scaled identity matrix. Figure 3 shows the BER performance of six systems versus $\mathrm{SNR}_{\mathrm{s}}$. It can be seen from Figure 3 that the NAF algorithm has the worst performance, since it does not exploit the channel knowledge available. Although both the ORO algorithm and the proposed MMSE (IPC) algorithm use a linear MMSE receiver at the destination node, the proposed algorithm has a better performance, since it jointly optimizes the source and relay matrices. We also observe from Figure 3 that as expected, the proposed optimal relay algorithm with the nonlinear MMSE-DFE receiver has the best BER performance. Note that although the algorithms in $[11,12]$ have a better BER performance compared with the proposed algorithms, the relay matrices developed by Toding et al. [11,12] do not satisfy the power constraints at each relay node, which is more relevant for practical relay communication systems.

In the second example, we study the effect of the number of relays to the system BER performance using the proposed algorithms. Figure 4 displays the system BER versus $\mathrm{SNR}_{\mathrm{s}}$ with $K=2,3$, and 5 . It can be seen that at $\mathrm{BER}=10^{-4}$, for both the linear MMSE-based optimal

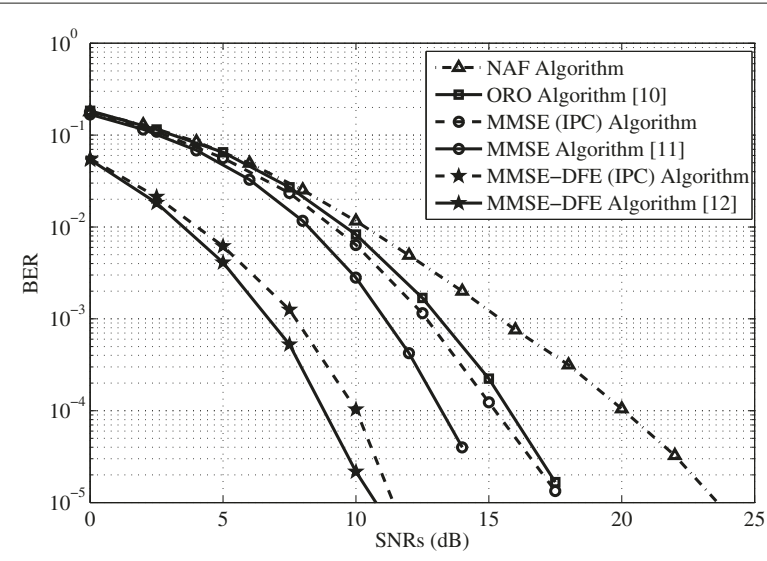

Figure 3 Example 1. BER versus $\mathrm{SNR}_{\mathrm{S}}$ with $K=3$.

relay system and the nonlinear MMSE-DFE-based optimal relay system, we can achieve approximately 5 -dB gain by increasing from $K=2$ to $K=5$. We would like to mention that although the nonlinear MMSE-DFE algorithm has an improved BER performance compared with the linear MMSE algorithm, the former system has a higher decoding complexity than the latter one. Such performance-complexity tradeoff is very useful for practical communication systems.

\section{Conclusions}

We have derived the optimal structure of the source precoding matrix and the relay amplifying matrices for parallel MIMO relay communication systems using linear MMSE receiver and nonlinear MMSE-DFE receiver at the destination node. The proposed source and relay matrices jointly diagonalize the source-relay-destination channel and minimize the MSE of the signal waveform estimation. Simulation results demonstrate that the proposed algorithms have improved BER performance compared with the existing techniques.

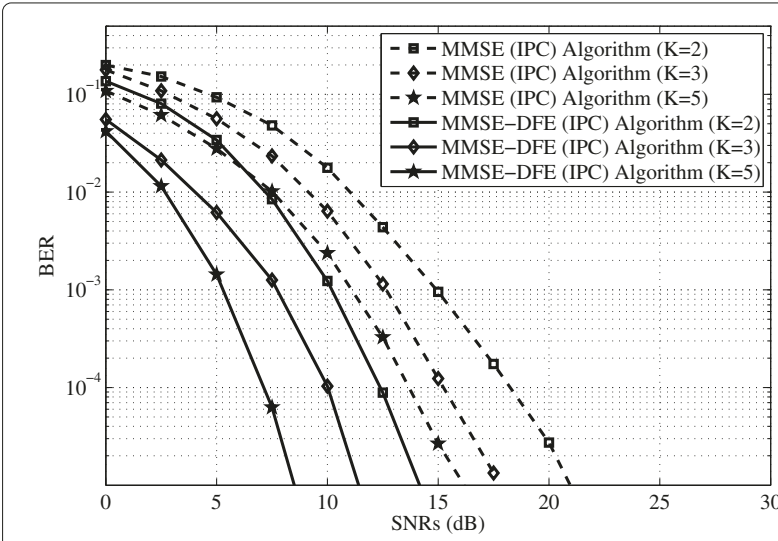

Figure 4 Example 2. BER versus $S N R_{S}$ with varying $K$. 


\section{Competing interests}

The authors declare that they have no competing interests.

\section{Acknowledgements}

This study was supported in part by the Australian Research Council's Discovery Projects funding scheme (project number DP110100736), the Higher Education Ministry of Indonesia (DIKTI), and the Paulus Christian University of Indonesia (UKI-Paulus) of Makassar, Indonesia (PhD scholarship of Apriana Toding).

Received: 25 January 2012 Accepted: 21 July 2012

Published: 16 August 2012

\section{References}

1. B Wang, J Zhang, A Høst-Madsen, On the capacity of MIMO relay channels. IEEE Trans. Inf. Theory. 51, 29-43 (2005)

2. $\mathrm{X}$ Tang, $\mathrm{Y}$ Hua, Optimal design of non-regenerative $\mathrm{MIMO}$ wireless relays. IEEE Trans. Wirel. Commun. 6, 1398-1407 (2007)

3. O Muñoz-Medina, J Vidal, A Agustín, Linear transceiver design in nonregenerative relays with channel state information. IEEE Trans. Signal Process. 55, 2593-2604 (2007)

4. W Guan, H Luo, Joint MMSE transceiver design in non-regenerative MIMO relay systems. IEEE Commun. Lett. 12, 517-519 (2008)

5. G Li, Y Wang, T Wu, J Huang, Joint linear filter design in multi-user cooperative non-regenerative MIMO relay systems. EURASIP J. Wirel. Commun. Netw. 2009, Article ID 670265 (2009)

6. Y Rong, Linear non-regenerative multicarrier MIMO relay communications based on MMSE criterion. IEEE Trans. Commun. 58, 1918-1923 (2010)

7. Z Fang, Y Hua, JC Koshy, Joint source and relay optimization for a non-regenerative MIMO relay. in Proc. IEEE Workshop Sensor Array Multi-Channel Signal Processing (Waltham, WA, 2006), pp. 239-243

8. Y Rong, X Tang, Y Hua, A unified framework for optimizing linear non-regenerative multicarrier MIMO relay communication systems. IEEE Trans. Signal Process. 57, 4837-4851 (2009)

9. Y Rong, Y Hua, Optimality of diagonalization of multi-hop MIMO relays. IEEE Trans. Wirel. Commun. 8, 6068-6077 (2009)

10. AS Behbahani, R Merched, AM Eltawil, Optimizations of a MIMO relay network. IEEE Trans. Signal Process. 56, 5062-5073 (2008)

11. A Toding, MRA Khandaker, Y Rong, Optimal joint source and relay beamforming for parallel MIMO relay networks. in Proc. 6th Int. Conf. Wireless Commun., Network. Mobile Comput. (Chengdu, China, 2010), pp. 23-25

12. A Toding, MRA Khandaker, Y Rong, Joint source and relay optimization for parallel MIMO relays using MMSE-DFE receiver. in Proc. 16th Asia-Pacific Conference on Communication (Auckland, New Zealand, November 1-3 2010), pp. 12-16

13. Y Rong, Optimal linear non-regenerative multi-hop MIMO relays with MMSE-DFE receiver at the destination. IEEE Trans. Wirel. Commun. $\mathbf{9}$ 2268-2279 (2010)

14. Y Jiang, $W$ Hager, J Li, The generalized triangular decomposition. Math. Comput. 77, 1037-1056 (2008)

15. AW Marshall, I Olkin, Inequalities: Theory of Majorization and Its Applications (Academic Press, New York, 1979)

\section{Submit your manuscript to a SpringerOpen ${ }^{\circ}$ journal and benefit from:}

- Convenient online submission

- Rigorous peer review

- Immediate publication on acceptance

- Open access: articles freely available online

- High visibility within the field

- Retaining the copyright to your article 